\title{
Expression and clinical significance of PD- L1, B7-H3, B7-H4 and TILs in human small cell lung Cancer (SCLC)
}

\author{
Daniel Carvajal-Hausdorf ${ }^{1,2}$, Mehmet Altan ${ }^{3,4}$, Vamsidhar Velcheti ${ }^{5}$, Scott N. Gettinger ${ }^{3}$, Roy S. Herbst ${ }^{3}$, \\ David L. Rimm ${ }^{1,3}$ and Kurt A. Schalper ${ }^{1,2,3^{*}}$
}

\begin{abstract}
Background: Small cell lung cancer (SCLC) accounts for 10-15\% of all lung malignancies and its prognosis is dismal. Although early studies have shown promising clinical activity of immune checkpoint blockers, the immune composition and expression of potentially actionable immunostimulatory targets in this malignancy are poorly understood.
\end{abstract}

Methods: Using multiplexed quantitative immunofluorescence (QIF), we measured the levels of 3 different B7 family ligands PD-L1, B7-H3, B7-H4 and major tumor infiltrating lymphocyte (TIL) subsets in 90 SCLC samples represented in tissue microarray format. Associations between the marker levels, clinicopathological variables and survival were studied.

Results: PD-L1 protein was detected in $7.3 \%, \mathrm{~B} 7-\mathrm{H} 3$ in $64.9 \%$ and $\mathrm{B} 7-\mathrm{H} 4$ in $2.6 \%$ of SCLC cases. The markers showed limited co-expression and were not associated with the level of TILs, age, gender and stage. Elevated B7-H4 was associated with shorter 5-year overall survival. The levels of CD3+, CD8+ and CD20+ TILs and the ratio of total/effector T-cells were significantly lower in SCLC than in non-small cell lung cancer. High levels of CD3+, but not CD8+ or CD20+ TILs were significantly associated with longer survival.

Conclusions: Taken together, our study indicate variable expression and clinical role of B7-family ligands in SCLC with predominant expression of the candidate target $\mathrm{B} 7-\mathrm{H} 3$ and the presence of a limited cytotoxic anti-tumor immune response. These results support the evaluation of B7-H3 blockers and/or pro-inflammatory therapies in SCLC.

\section{Background}

Small cell lung cancer (SCLC) accounts for approximately $10-15 \%$ of all lung carcinomas and comprise high-grade neuroendocrine tumors with aggressive clinical course and prominent association with tobacco use [1-3]. To date, there are limited therapeutic options and the prognosis is ominous with 5-year survival rates of only around 3-6\% for extensive stage SCLC [1]. Molecular characterization of SCLC has revealed an extremely high nonsynonymous mutational rate and the presence of deleterious variants in the tumor suppressor genes TP53

\footnotetext{
* Correspondence: kurt.schalper@yale.edu

'Department of Pathology, Yale School of Medicine, New Haven, USA

${ }^{2}$ Anatomia Patologica, Clinica Alemana, Facultad de Medicina Universidad del Desarrollo, Santiago, Chile

Full list of author information is available at the end of the article
}

and RB1 virtually in all cases [3, 4]. In addition, SCLCs contain relatively low frequency of mutations in actionable oncogenes, limiting the therapeutic options [5].

Immunostimulatory therapies blocking the PD-1 axis produce prominent and lasting clinical responses in nearly $20 \%$ of non-small cell carcinomas (NSCLC), the most common form of lung cancer [6-9]. The clinical benefit to PD-1 axis blockers is associated with tumor PD-L1 expression, pre-existing anti-tumor immune response and increased tumor mutational burden [6, 8-11]. Although preliminary data from ongoing trials using antagonistic PD-1 and CTLA-4 antibodies in heavily pre-treated SCLCs suggests limited activity of monotherapy regimens, combination PD-1/CTLA-4 immune checkpoint blockade show encouraging results with objective responses in up to $\sim 30 \%$ of cases $[12,13]$. Despite these results, little is 
known about the immune composition of SCLC and most studies characterizing immune cells or targets have used qualitative/subjective methods. Identification of dominant immune cell populations and/or expression of candidate immunotherapy targets in this tumor could support optimal design and interpretation of clinical trials.

PD-L1 protein expression has been found in a highly variable proportion of SCLC ranging from $0 \%$ in one study including 61 samples [14] to $71.6 \%$ in another study with 102 cases [15]. The biological determinants for this discrepancy remain unknown but are likely due to technical differences or limitations of the methods used. Here, we used validated assays and multiplexed quantitative immunofluorescence (QIF) to objectively measure and assess the clinical impact of PD-L1, B7-H3, B7-H4 and major TIL subpopulations in human SCLCs.

\section{Methods}

\section{Patients, cohorts and tissue microarrays}

Samples from a retrospectively collected SCLC cohort from Yale University represented in 2 tissue microarrays (TMAs) (YTMA57 and YTMA259) totaling 90 cases were used. Detailed clinico-pathological characteristics of the cohorts were collected form surgical pathology reports and clinical records. TMAs were prepared using $0.6 \mathrm{~mm}$ tissue cores, each in 2-fold redundancy using standard procedures [16, 17]. The actual number of samples analyzed for each marker is lower than the total samples in the cohort due to unavoidable loss of tissue, absence or limited tumor cells in some spots as is commonly seen in TMA studies or incomplete clinicopathologic annotation. All tissue was used after approval from the Yale Human Investigation Committee protocols \#9505008219 and \#1608018220, which approved the patient consent forms or in some cases waiver of consent.

\section{Multiplexed quantitative immunofluorescence (QIF)}

We measured the levels of PD-L1 (E1L3N, Cell Signaling technology), B7-H3 (D9M2L, Cell Signaling Technology), B7-H4 (D1M8I, Cell Signaling Technology), CD3 (clone E272, Novus Biologicals), CD8 (clone C8/144B, DAKO), CD20 (clone L26, DAKO) and pancytokeratin (AE1/AE3, DAKO) using QIF in TMA slides containing the cohort cases. PD-L1, B7-H3 and B7-H4 were stained in serial sections from the TMA blocks using a previously described protocol with simultaneous detection of cytokeratin and 4',6-diamidino-2-phenylindole (DAPI) $[18,19]$. Briefly, antigen retrieval was with citrate buffer $\mathrm{pH} 6.0$ for $20 \mathrm{~min}$ at $97^{\circ} \mathrm{C}$ in a pressure-boiling container and blocking was performed with $0.3 \%$ bovine serum albumin in $0.05 \%$ Tween solution for $30 \mathrm{~min}$. Primary antibodies were incubated overnight using a dilution of 1:1600 for PD-L1, 1:500 for B7-H3 and
1:200 for B7-H4. Stringent validation and optimization of these assays using cell line transfectants and endogenous human tissue controls has been reported by our group [18, 20,21]. Secondary antibody for cytokeratin was Alexa 546-conjugated goat anti-mouse or anti-rabbit (Invitrogen Molecular Probes, Eugene, OR, USA). Cyanine 5 (Cy5) directly conjugated to tyramide (FP1117; Perkin-Elmer) at a 1:50 dilution was used for target antibody detection.

CD3, CD8, CD20 and cytokeratin were simultaneously stained using a sequential staining protocol, as previously described [16, 20,22]. Briefly, TMA sections were deparaffinized and subjected to antigen retrieval using $\mathrm{pH}=8.0$ EDTA buffer (Sigma-Aldrich, St Louis, MO, USA) and boiled for $20 \mathrm{~min}$ at $97^{\circ} \mathrm{C}$ in a pressureboiling container (PT module, Lab Vision, Thermo Scientific, Waltham, MA, USA). Slides were then incubated with dual endogenous peroxidase block (DAKO $\#$ \#2003, Carpinteria, CA, USA) for $10 \mathrm{~min}$ at room temperature and subsequently with a blocking solution containing $0.3 \%$ bovine serum albumin in $0.05 \%$ Tween solution for $30 \mathrm{~min}$. Residual horseradish peroxidase activity between incubations with secondary antibodies was eliminated by exposing the slides twice for $7 \mathrm{~min}$ to a solution containing benzoic hydrazide $(100 \mathrm{mM})$ and hydrogen peroxide $(50 \mathrm{mM})$ in PBS. Isotype specific, fluorophore-conjugated secondary antibodies were used for signal detection and nuclei were highlighted using DAPI.

\section{Fluorescence signal quantification and cases stratification}

Quantitative measurement of the fluorescent signal was performed using the $\mathrm{AQUA}^{\circ}$ method of QIF, as previously reported $[18,20,23]$. Briefly, the QIF score of each fluorescence channel was calculated by dividing the target marker pixel intensities by the area of the desired compartment. Scores were normalized to the exposure time and bit depth at which the images were captured, allowing scores collected at different exposure times to be comparable. The immune target scores and TIL markers considered the signal detected in the whole tissue compartment using an adjusted DAPI mask. Cases were considered as target expressers when the QIF score was above the signal detection threshold determined using the negative control preparations and visual inspection. For stratification, the marker levels were classified as high/low using the top 25-percentile of the cohort scores as stratification cut-point.

\section{Statistical analyses}

QIF signal differences between groups were analyzed using t-test for continuous variables and chi-square test for categorical variables. Linear regression coefficients 
were calculated to determine the association between continuous scores. Survival analysis based on marker expression was performed using Kaplan-Meier analyses with log rank test and overall survival as endpoint. Statistical significance was considered at $P<0.05$ and analyses were performed using $\mathrm{JMP}^{\circ}$ Pro software (version 9.0.0, 2010, SAS Institute Inc.) and GraphPad Prism v6.0 for Windows (GraphPad Software, Inc). All statistical tests were two-sided.

\section{Results}

\section{Expression of PD-L1, B7-H3, B7-H4 and TILs in SCLC}

We previously validated and optimized assays for detection of PD-L1, B7-H3, B7-H4 and TIL markers using formalin-fixed, paraffin-embedded (FFPE) preparations from human tissue samples and cell line transfectants [17, 20-23]. As expected for SCLC, positive staining for cytokeratin was focal and frequently showed a perinuclear dot-like staining pattern (Fig. 1). PD-L1 and B7-H3 were predominantly recognized in tumor cells with cytoplasmic and membranous staining (Fig. 1a). Prominent B7-H4 positivity was infrequently recognized and showed relatively low signal with a focal staining pattern. Expression of TIL markers showed predominance of CD3+ T-cell staining with CD8+ and $\mathrm{CD} 20+$ cells displaying low levels in the cohort. Representative examples from cases with prominent CD3+ TILs or CD20+ B-cell infiltrates are shown in Fig. 1b.

Using the visual detection threshold by pathologists-based analysis, we detected tumor-cell PD-L1, B7-H3 and B7-H4 in 7.3, 64.9 and $2.6 \%$ of cases in the cohort (Fig. 2). In the QIF analysis, PD-L1 and B7-H4 show relatively low scores, while B7-H3 had a wider range with cases displaying prominently higher signal. Overall, the levels of $\mathrm{B} 7-\mathrm{H} 3$ were 2.3 fold higher than PD-L1 (mean QIF score 894 vs $386, P=0.02$ ) and 5.8 fold higher than B7-H4 (mean QIF score 894 vs 155 , $P<0.001)$. Notably, the levels of the targets showed limited correlation with PD-L1 and B7-H3 showing minimal co-expression consistent with a mutually exclusive expression pattern (Fig. 3a).

Expression of the TIL markers showed a wide range and continuous score distribution with $16 \%$ of cases displaying undetectable B and T-cell infiltration (Fig. 4). CD3 showed the highest dynamic range of all markers and was detected in $94 \%$ of specimens. CD8+ T-cell infiltration was identified in $67 \%$ of cases and CD20+ B-lymphocyte signal was seen only in $11 \%$ of cases.

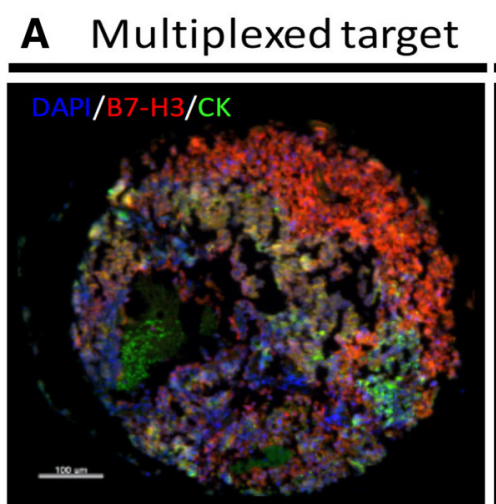

\section{B Multiplexed TILs}

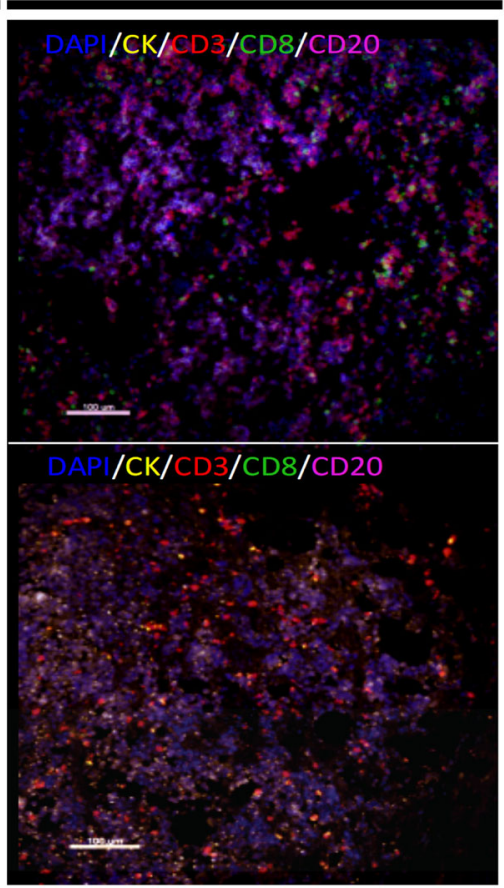

Fig. 1 Detection of immune targets and TILS in SCLC using multiplex quantitative fluorescence. a Representative fluorescence pictures showing B7-H3 (upper panel) and PD-L1 (lower panel) protein expression in SCLC. The target signal (red fluorescence) is predominantly located in tumor cells. b Representative fluorescence pictures showing the signal for DAPI (blue), cytokeratin (CK, green), CD3 (red), CD8 (green) and CD20 (magenta) staining in SCLC. Bar $=100 \mathrm{um}$ 


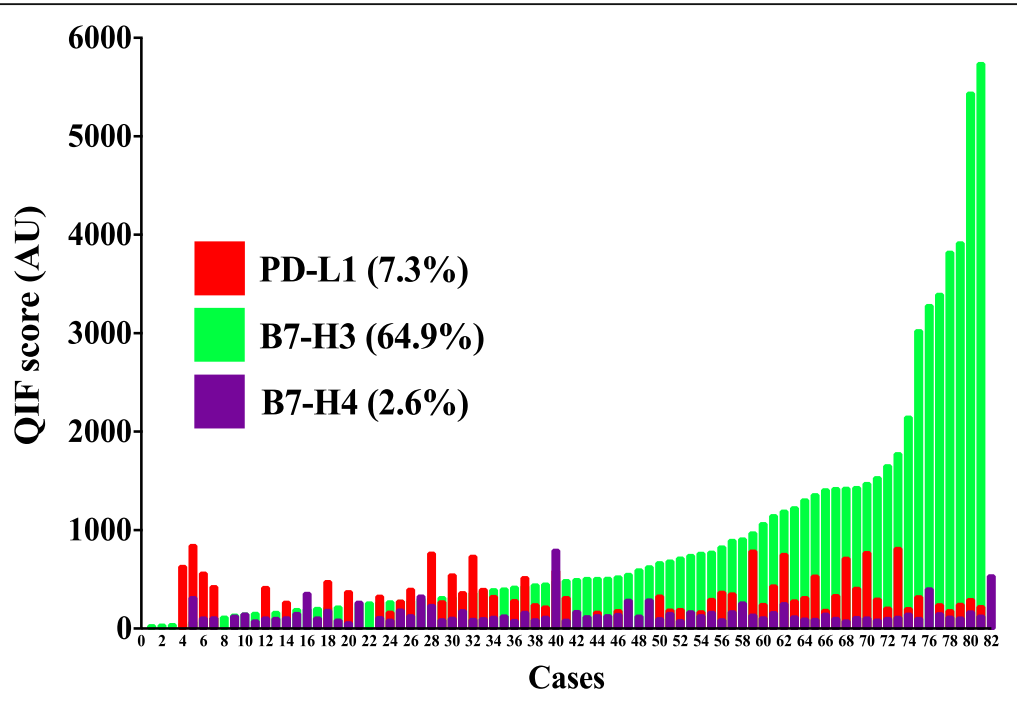

Fig. 2 Levels of different immune targets in SCLC. Distribution of PD-L1 (red), B7-H3 (green) and B7-H4 (magenta) QIF scores in SCLCs from Yale. The frequency of expression for each marker is indicated in parenthesis. The cut-point used to define expression was the signal detection threshold. $\mathrm{AU}=$ Arbitrary units of fluorescence

\section{Tumor immune infiltration of SCLC and comparison with} NSCLC

To evaluate the TIL scores of SCLCs in the context of other lung cancer subtypes, we compared the marker levels with those obtained in retrospective cohorts of lung adenocarcinomas (LADC) and lung squamous cell carcinomas (LSCC) measured using the same assay and analysis platform [22]. As shown in Fig. 5a, SCLCs showed significantly lower levels of all TIL markers than LADC and LSCC $(P=0.01-P<0.0001)$. The most prominent difference was in CD8 level that was 5.4 fold lower than in LADC and 6-fold lower than in LSCC. Notably, the CD3/CD8 ratio was also prominently lower in SCLC than in the major NSCLC subsets, suggesting the presence of a less cytotoxic $\mathrm{T}$-cell profile in this malignancy (Fig. 5b, mean CD3/CD8 ratio of 0.37 vs 0.63 in LADC and 0.62 in LSCC, $P<0.001)$.

\section{Association of the markers with clinicopathologic variables and survival}

Elevated expression of PD-L1, B7-H3 or B7-H4 (scores within the top signal quartile) were not significantly associated with major clinicopathologic variables or TIL markers in the cohort (Table 1). As expected, the levels of CD3 were positively associated with $\mathrm{CD} 8$, but there was no relationship between CD3 or CD8 and CD20 in the tumors. High levels of CD20+ B-cells were more commonly seen in samples from female patients (14 of 23 [37.8\%] vs 6 of 38 [13.6\%], $P=0.01)$. High PD-L1 or B7-H3 protein levels were not significantly associated with 5-year overall survival (Fig. 6a-b). However, elevated expression of B7-H4 was associated with shorter survival in the cohort (Fig. 6c, log-rank $P=0.05$ ). In addition, increased expression of the pan T-cell marker CD3- but not of CD8 or CD20 was significantly associated with longer overall survival (log-rank $P=0.03$, Fig. $6 \mathrm{~d}-\mathrm{f}$ ).
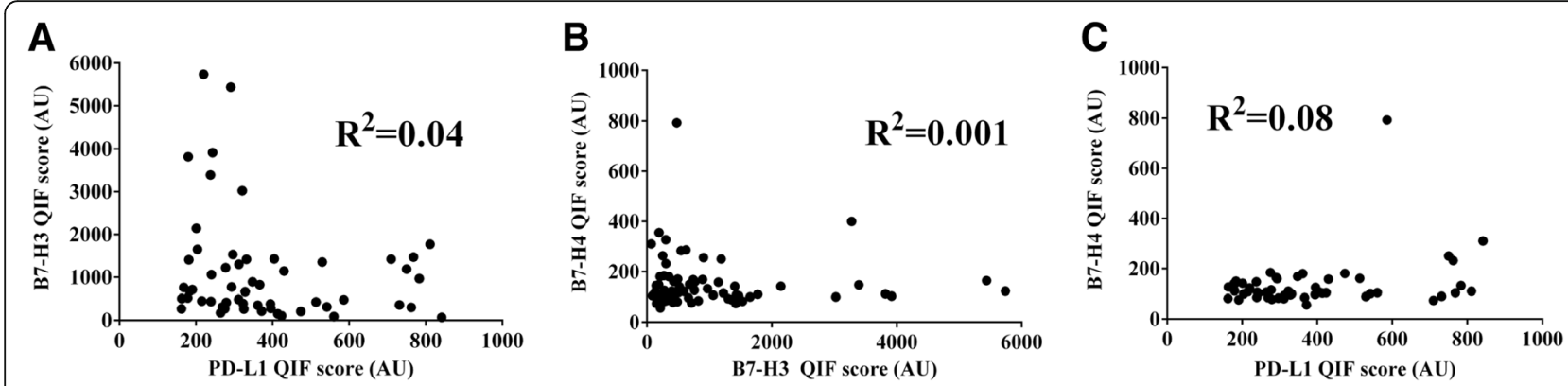

Fig. 3 PD-L1, B7-H3 and B7-H4 are infrequently co-expressed in SCLC. A-C) Histograms showing the levels of PD-L1, B7-H3 and B7-H4 protein in small cell lung carcinomas from the Yale cohort. The linear regression coefficients $\left(R^{2}\right)$ of the scores between each marker pair are indicated within the charts 
Distribution of CD3, CD8 and CD20

scores in Yale SCLC Cohort

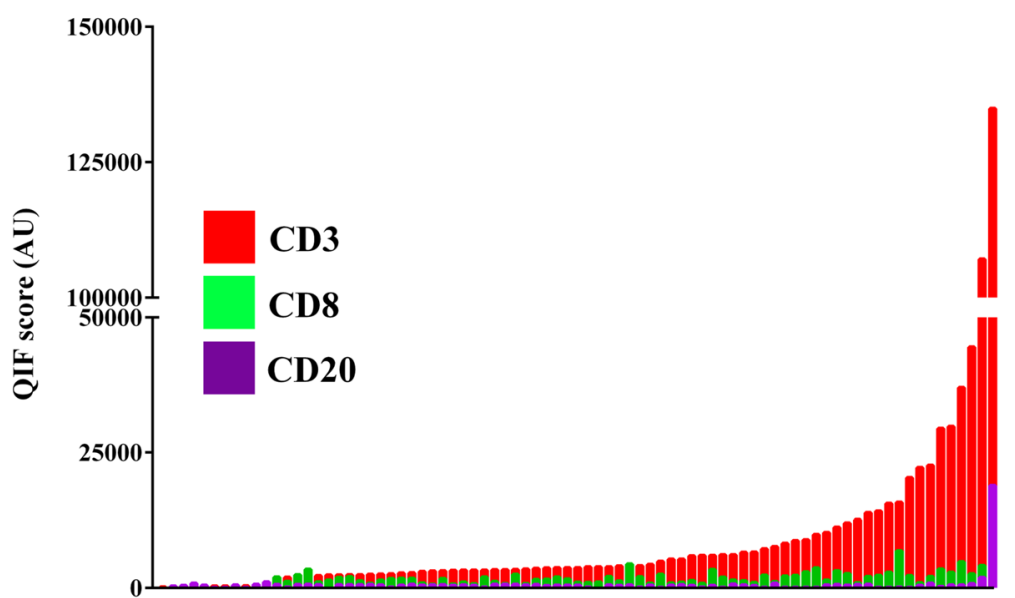

Cases

Fig. 4 Levels of TIL subpopulations in SCLC. Distribution of CD3 (red), CD8 (green) and CD20 (magenta) QIF scores in SCLCs from the Yale cohort. Cases were stratified using the median score of each marker as stratification cut-point. AU = Arbitrary units of fluorescence

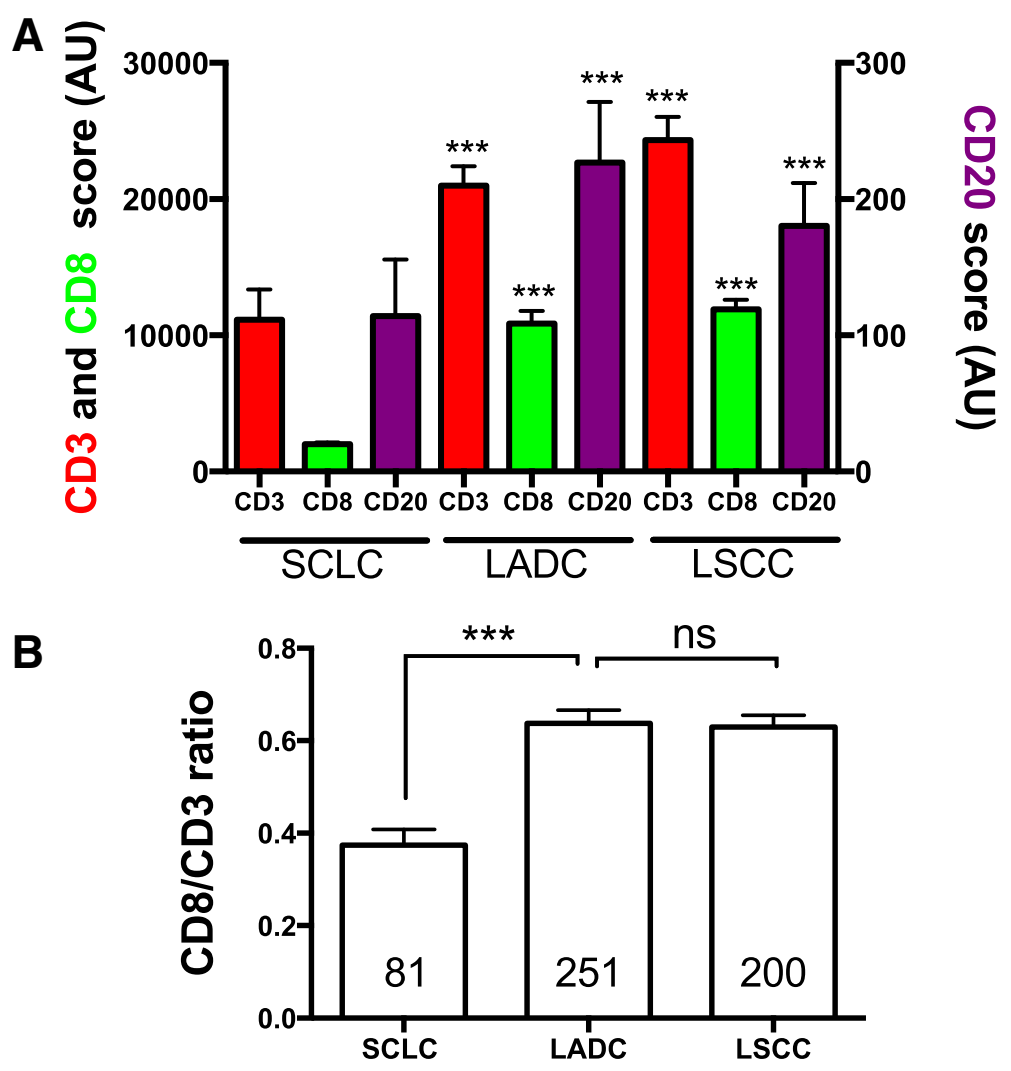

Fig. 5 Levels of TIL subpopulations in SCLC and major NSCLC subtypes. a Chart showing the levels of CD3 (red), CD8 (green) and CD20 (magenta) in SCLC (left), primary lung adenocarcinomas (LADC, center) and lung squamous cell carcinomas (LSCC, right). Each bar depicts the median +/- SEM. The levels of TILS in NSCLC subtypes were obtained previously using the same multiplexing protocol [22]. b Chart showing the ratio of CD8/CD3 signal in SCLCS (left), LADCs (center) and LSCCs (right). The number of cases is indicated within each bar. ${ }^{* * *}=P<0.001 ; \mathrm{ns}=$ not significant. $\mathrm{AU}=$ Arbitrary units of fluorescence 
Table 1 Association of PD-L1, B7-H3, B7-H4 and TIL subsets with major clinico-pathological characteristics and TILs in SCLC

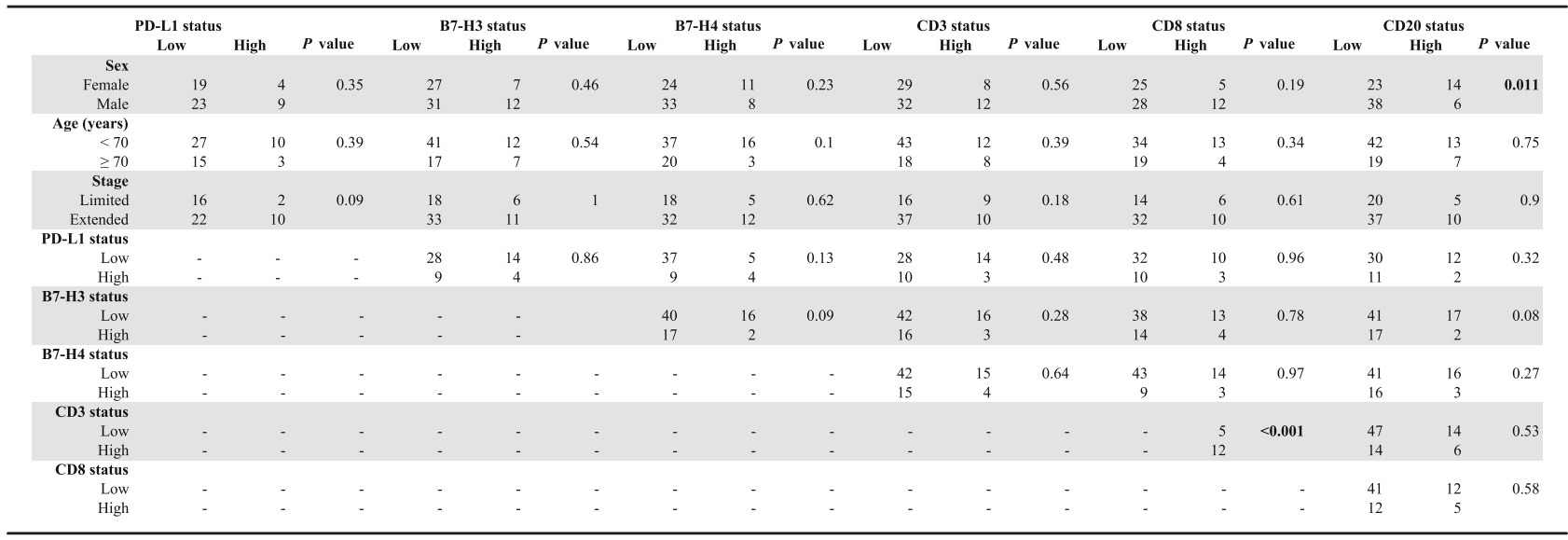

\section{Discussion and conclusions}

Using multiplexed tissue analysis, we have objectively measured 3 different B7-family member ligands and TIL subsets in a sizable collection of human SCLCs. We found relatively low levels of PD-L1, B7-H4 and TILs; but prominent expression of $\mathrm{B} 7-\mathrm{H} 3$ protein. In addition, we found a previously unrecognized negative prognostic role of $\mathrm{B} 7-\mathrm{H} 4$ and a positive prognostic effect of $\mathrm{CD} 3+$ TILs in this malignancy. Taken together, our data support that SCLC is a relatively "immune-cold" tumor and suggests the presence of prominent immune regulatory mechanisms. Elevated expression of B7-H3 could mediate immune evasion in SCLC and represent a therapeutic opportunity.

Diverse studies have interrogated the expression of PD-L1 by chromogenic immunohistochemistry (IHC) in SCLC and have reported highly variable results ranging $0-71.6 \%[14,15,24]$. These differences could be explained using different IHC assays, analysis platforms and stratification cut-points. One study showing $71.6 \%$ PD-L1 expression used a commercial rabbit monoclonal antibody (Abcam, Cambridge, UK) with $5 \%$ positive

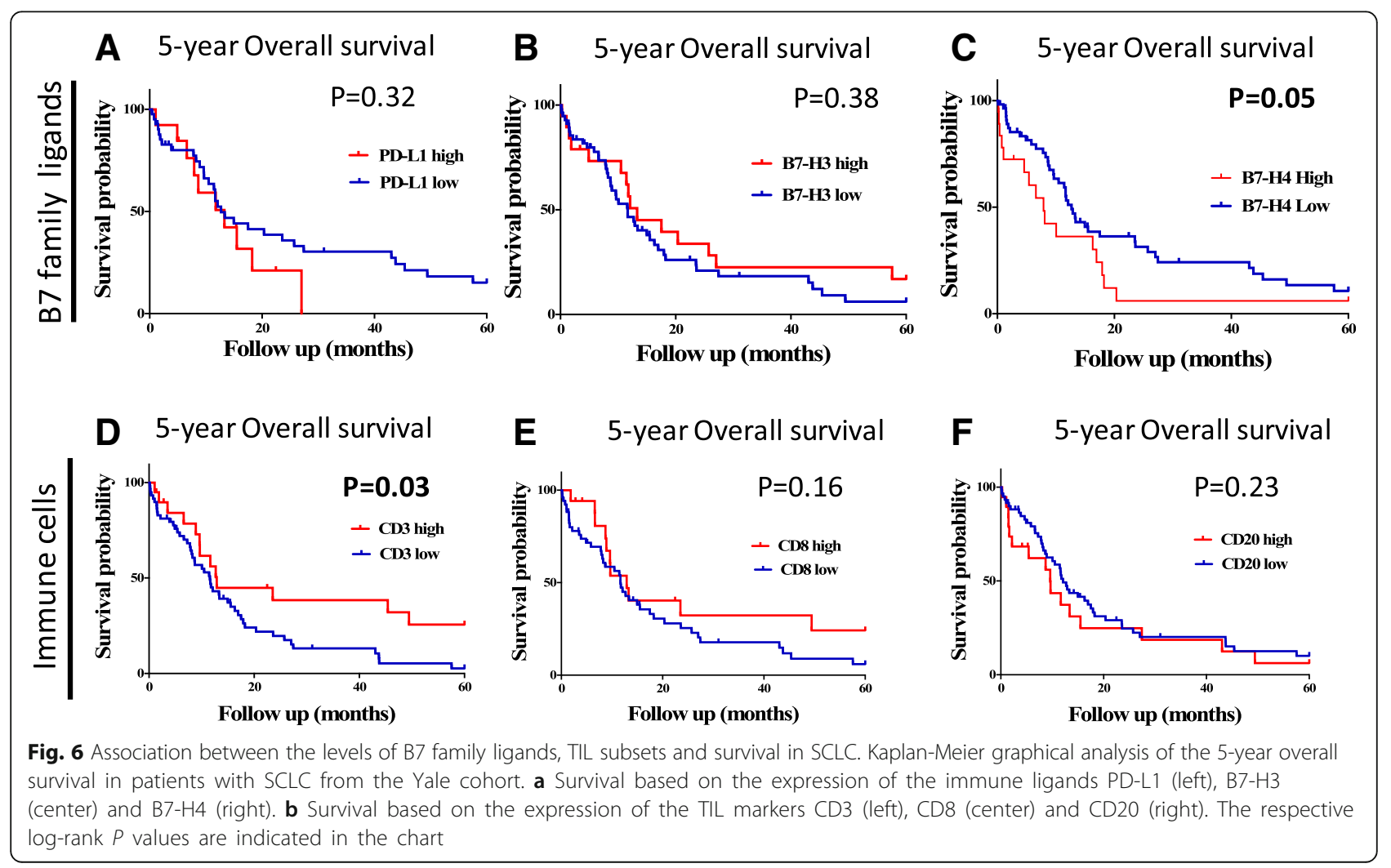


tumor cell as cut-point, but did not specify the clone name and validation status [15]. Two other studies using the validated antibody clone E1L3N [25] and semiquantitative scoring reported expression frequencies of $0 \%$ in tumor cells $(0 / 94$ cases) with $18.5 \%$ in stromal/immune cells (17/92 cases) [14]; and 5.8\% overall PD-L1 expression (4 of 69 cases) [24]. The latter results are similar to our study showing infrequent expression of PD-L1 in SCLC.

Although anti-tumor activity of PD-1 blocking agents has been shown in recurrent SCLC [12, 13], the predictive value of tumor PD-L1 expression in this malignancy is unknown. Future studies directly comparing the clinical benefit of patients with PD-L1 positive and negative SCLCs will be required to clarify this.

Another finding was the common/high expression of $\mathrm{B} 7-\mathrm{H} 3$ and relatively low expression of $\mathrm{B} 7-\mathrm{H} 4$ in the cohort. To the best of our knowledge, this is the first report about the expression of these targets in SCLC. Interestingly, both markers showed minimal coexpression and low association with PD-L1 suggesting a non-redundant/exclusive expression pattern. A similar finding was recently communicated by our group in NSCLC [21]. In SCLCs the expression of PD-L1, B7-H3 and $\mathrm{B} 7-\mathrm{H} 4$ was not associated with the level of CD3, CD8 or CD20+ TILs. However, elevated expression of B7-H4 was significantly associated with worse overall survival supporting a role of this marker in SCLC progression.

Targeting B7-H3 is currently being evaluated as anti-cancer immunostimulatory strategy in preclinical models and in early phase clinical trials $[26,27]$. Enoblituzumab (MGA271, Macrogenics) is an Fc-optimized monoclonal antibody to selectively target $\mathrm{B} 7-\mathrm{H} 3$ and is currently in phase 1 studies alone or in combination with PD-1/CTLA-4 inhibitors (NCT02475213, NCT01391143 and NCT02381314). Further understanding of the modulation of B7-H3 expression, identification of its cognate receptor(s) and immuno-modulatory role in cancer will be key to support further clinical development of this pathway.

Our data show that SCLCs display relatively low $\mathrm{T}$ - and B-cell infiltration despite being traditionally associated with prominent tobacco exposure, high mutational load and production of neuroendocrine antibodies mediating autoimmune paraneoplastic syndromes [3, 4, 28], In addition, SCLCs have a low total/effector T-cell ratio and limited association between TIL levels and survival. This supports a limited adaptive anti-tumor response in most SCLCs and suggests the presence of potent tolerogenic mechanisms in this malignancy. Possible mechanisms involved in immune evasion are currently unknown but may include an altered tumor microvasculature, epigenetic silencing of immunogenic tumor epitopes, metabolic competition between tumor and immune cells and expression of multiple potent immune suppressive targets/pathways [28]. Additional studies will be required to explore these possibilities. Notably and different to other tumor types [29,30], only CD3+ but not CD8+ TILs were prognostic in SCLCs. A lack of prognostic value of CD8+ TILs as measured by chromogenic IHC and semi-quantitative scoring was also recently reported in a retrospective cohort of 66 stage I-III lung SCLCs [31]. The limited prognostic value of cytotoxic CD8+ TILs in SCLC could be at least partially explained by the relatively low levels of this immune cell subset. The positive prognostic effect of CD3 could be due to a higher dynamic range of this marker and to the contribution to this score of additional non-cytotoxic CD3+ immune cell populations such as CD4+ TILs and NKT cells.

Our study has limitations. The evaluation of cases was performed using TMAs with possible over/under-representation of the markers due to evaluation of relatively small tumor areas. In addition, tumor tissue was obtained from a single tumor location, limiting the representation of additional lesions not sampled during the diagnostic workup. However, diverse reports measuring immune markers using TMAs from individual tumor lesions have shown consistent results and significant association with clinicopathologic features and outcome supporting the value of this approach [16, 18, 20, 22, 25]. Finally, the cut-points used for marker stratification were based on the relative abundance of the protein signal within the cohort and should be considered as exploratory. Additional studies using independent SCLC collections will be required to validate optimal marker stratification strategies in this disease.

In summary, we have quantitatively measured the expression of 3 different B7-family ligands and major TIL populations in human SCLC. Our data indicate variable expression of the markers with predominance of the candidate immunostimulatory target $\mathrm{B} 7-\mathrm{H} 3$; and the presence of a limited cytotoxic anti-tumor immune response in this malignancy.

\section{Abbreviations \\ DAPI: 4',6-diamidino-2-phenylindole; FFPE: Formalin-fixed, paraffin- embedded; IHC: Immunohistochemistry; LADC: Lung adenocarcinoma; LSCC: Lung squamous cell carcinoma; NSCLC: Non-small cell lung cancer; QIF: Quantitative immunofluorescence; SCLC: Small cell lung cancer; TILS: Tumor-infiltrating lymphocytes; TMA: Tissue microarray \\ Acknowledgements \\ Not applicable. \\ Funding \\ FONDECYT Grant No. 1150731, Lung Cancer Research Foundation (LCRF), Yale SPORE in Lung Cancer (P50CA196530), Department of Defense-Lung Cancer Research Program Career Development Award (W81XWH-16-1-0160), Stand Up To Cancer - American Cancer Society Lung Cancer Dream Team Translational Research Grants (SU2C-AACR-DT17-15 and SU2C-AACR-DT22- 17), Yale Cancer Center Support Grant (P30CA016359) and sponsored research by Navigate BioPharma.}




\section{Availability of data and materials}

De-identified datasets used and/or analyzed during the current study are available from the corresponding author upon reasonable request.

\section{Authors' contributions}

Study conception and design: DEC, KAS. In vitro assays: DEC, KAS. Staining of clinical cases and image analysis: DEC, KAS. Statistical analyses: DEC, KAS. Drafting of manuscript: DEC, KAS. Critical revision of manuscript: all authors. Supervision \& securing of study resources: KAS. All authors read and approved the final manuscript.

\section{Ethics approval and consent to participate}

All tissue was used after approval from the Yale Human Investigation Committee protocols \#9505008219 and \#1608018220, which approved the patient consent forms or in some cases waiver of consent.

\section{Consent for publication}

Not applicable.

\section{Competing interests}

Kurt Schalper: Consultant or advisor for Celgene, Moderna Therapeutics, Shattuck Labs, AstraZeneca, Pierre-Fabre and Abbvie. Research funding by Navigate Biopharma, Vasculox/Tioma, Tesaro, Takeda, Moderna Therapeutics, Surface Oncology, Pierre-Fabre, Merck and Bristol-Myers Squibb.

David Rimm: Consultant or advisor for Amgen, AstraZeneca, Agendia, Biocept, BMS, Cell Signaling Technology, Cepheid, Daiichi Sankyo, GSK, InVicro/Konica/ Minolta, Merck, NanoString, Perkin Elmer, PAIGE.Al, and Ultivue. Equity holder in PixelGear (start-up company related to direct tissue imaging) Research funding from AstraZeneca, Cepheid, Navigate/Novartis, NextCure, Lilly, Ultivue, Ventana and Perkin Elmer/Akoya.

Mehmet Altan: Research funding from BMS and Lilly.

Vamsidhar Velcheti: Consultant or advisory role for Genentech, BMS, AstraZeneca, Merck, Nektar therapeutics, Reddy Labs, Celgene, Foundation Medicine and Takeda Oncology.

Roy Herbst: Consultant or advisory role for Abbvie Pharmaceuticals, AstraZeneca, Biodesix, Bristol-Myers Squibb , Eli Lilly and Company, EMD Serono, Genentech/Roche, Heat Biologics, Loxo Oncology, Merck and Company, Nektar, NextCure, Novartis, Pfizer, Sanofi, Seattle Genetics, Shire PLC, Spectrum Pharmaceuticals, Symphogen, Tesaro, Neon Therapeutics, Infinity Pharmaceuticals. Research support from AstraZeneca, Eli Lilly and Company, Merck and Company. Member of the board of directors (nonexecutive/ independent) for Junshi Pharmaceuticals.

\section{Publisher's Note}

Springer Nature remains neutral with regard to jurisdictional claims in published maps and institutional affiliations.

\section{Author details \\ 'Department of Pathology, Yale School of Medicine, New Haven, USA. ${ }^{2}$ Anatomia Patologica, Clinica Alemana, Facultad de Medicina Universidad del Desarrollo, Santiago, Chile. ${ }^{3}$ Medical Oncology, Yale School of Medicine and Yale Cancer Center, 333 Cedar St. FMP117, New Haven, CT 06520-8023, USA. ${ }^{4}$ Thoracic Oncology, MD Anderson Cancer Center, Camden, USA. ${ }^{5}$ Thoracic Oncology, New York University, New York, USA.}

\section{Received: 6 November 2018 Accepted: 20 February 2019}

\section{Published online: 08 March 2019}

\section{References}

1. Altan M, Chiang AC. Management of Small Cell Lung Cancer: Progress and updates. Cancer J. 2015;21:425-33.

2. Koinis F, Kotsakis A, Georgoulias V. Small cell lung cancer (SCLC): no treatment advances in recent years. Transl Lung Cancer Res. 2016:5:39-50.

3. Peifer M, Fernández-Cuesta L, Sos ML, George J, Seidel D, Kasper LH, et al. Integrative genome analyses identify key somatic driver mutations of smallcell lung cancer. Nat Genet. 2012;44:1104-10.

4. George J, Lim JS, Jang SJ, Cun Y, Ozretia L, Kong G, et al. Comprehensive genomic profiles of small cell lung cancer. Nature. 2015;524:47-53.

5. Arcaro A. Targeted therapies for small cell lung cancer: Where do we stand? Crit Rev Oncol Hematol. 2015;95(2):154-64.
6. Garon EB, Rizvi NA, Hui R, Leighl N, Balmanoukian AS, Eder JP, et al. Pembrolizumab for the treatment of non-small-cell lung Cancer. N Engl J med. 2015;372:2018-28.

7. Borghaei H, Paz-Ares L, Horn L, Spigel DR, Steins M, Ready NE, et al. Nivolumab versus docetaxel in advanced nonsquamous non-small-cell lung Cancer. N Engl J Med. 2015;373:1627-39.

8. Herbst RS, Baas P, Kim D-W, Felip E, Pérez-Gracia JL, Han J-Y, et al. Pembrolizumab versus docetaxel for previously treated, PD-L1-positive, advanced non-small-cell lung cancer (KEYNOTE-010): a randomised controlled trial. Lancet (London, England). 2016;387:1540-50.

9. Herbst RS, Soria JC, Kowanetz M, Fine GD, Hamid O, Gordon MS, et al. Predictive correlates of response to the anti-PD-L1 antibody MPDL3280A in cancer patients. Nature. 2014;515:563-7.

10. Rizvi NA, Hellmann MD, Snyder A, Kvistborg P, Makarov V, Havel JJ, et al. Cancer immunology. Mutational landscape determines sensitivity to PD-1 blockade in non-small cell lung cancer. Science. 2015;348:124-8.

11. McGranahan N, Furness AJS, Rosenthal R, Ramskov S, Lyngaa R, Saini SK, et al. Clonal neoantigens elicit T cell immunoreactivity and sensitivity to immune checkpoint blockade. Science. 2016;351:1463-9.

12. Antonia SJ, López-Martin JA, Bendell J, Ott PA, Taylor M, Eder JP, et al. Nivolumab alone and nivolumab plus ipilimumab in recurrent small-cell lung cancer (CheckMate 032): a multicentre, open-label, phase 1/2 trial. Lancet Oncol. 2016;17:883-95.

13. Ott PA, Elez E, Hiret S, Kim DW, Morosky A, Saraf S, et al. Pembrolizumab in patients with extensive-stage small-cell lung cancer: results from the phase Ib KEYNOTE-028 study. J Clin Oncol. 2017;35:3823-9.

14. Schultheis AM, Scheel AH, Ozretić L, George J, Thomas RK, Hagemann T, et al. PD-L1 expression in small cell neuroendocrine carcinomas. Eur J Cancer. 2015;51:421-6.

15. Ishii H, Azuma K, Kawahara A, Yamada K, Imamura Y, Tokito T, et al. Significance of programmed cell death-ligand 1 expression and its association with survival in patients with small cell lung cancer. J Thorac Oncol. 2015;10:426-30.

16. Carvajal-Hausdorf DE, Schalper KA, Neumeister VM, Rimm DL. Quantitative measurement of cancer tissue biomarkers in the lab and in the clinic. Lab Investig. 2015;95:385-96.

17. Velcheti V, Schalper KA, Carvajal DE, Anagnostou VK, Syrigos KN, Sznol M, et al. Programmed death ligand-1 expression in non-small cell lung cancer. Lab Invest. 2014;94:107-16.

18. Velcheti V, Schalper KA, Carvajal DE, Anagnostou VK, Syrigos KN, Sznol M, et al. Programmed death ligand-1 expression in non-small cell lung cancer. Lab Investig. 2013;00:1-10.

19. Gettinger SN, Choi J, Mani N, Sanmamed MF, Datar I, Sowell R, Du VY, Kaftan E, Goldberg S, Dong W, Zelterman D, Politi K, Kavathas P, Kaech S, Yu $X$, Zhao H, Schlessinger J, Lifton R, Rimm DL, Chen L, Herbst RS, Schalper KA..A dormant TIL phenotype defines non-small cell lung carcinomas sensitive to immune checkpoint blockers. Nat Commun. 2018;9(1):3196.

20. Schalper KA, Carvajal-Hausdorf D, McLaughlin J, Altan M, Velcheti V, Gaule P, et al. Differential expression and significance of PD-L1, IDO-1, and B7-H4 in human lung cancer. Clin Cancer Res. 2017;23:370-8.

21. Altan M, Pelekanou V, Schalper KA, Toki M, Gaule P, Syrigos K, et al. B7-H3 expression in NSCLC and its association with B7-H4, PD-L1 and tumorinfiltrating lymphocytes. Clin Cancer Res. 2017;23:5202-9.

22. Schalper KA, Brown J, Carvajal-Hausdorf D, McLaughlin J, Velcheti V, Syrigos $\mathrm{KN}$, et al. Objective Measurement and Clinical Significance of TILs in NonSmall Cell Lung Cancer. J Natl Cancer Inst. 2015;107:dju435.

23. McLaughlin J, Han G, Schalper KA, Carvajal-Hausdorf D, Pelakanou V, Rehman J, et al. Quantitative assessment of the heterogeneity of PD-L1 expression in non-small-cell lung Cancer. JAMA Oncol. 2015;2:1-9.

24. Tsuruoka K, Horinouchi $H$, Goto $Y$, Kanda S, Fujiwara Y, Nokihara $H$, et al. PD-L1 expression in neuroendocrine tumors of the lung. Lung Cancer. 2017;108:115-20.

25. Wimberly H, Brown JR, Schalper K, Haack H, Silver MR, Nixon C, et al. PD-L1 expression correlates with tumor-infiltrating lymphocytes and response to neoadjuvant chemotherapy in breast Cancer. Cancer Immunol Res. 2015;3:326-32.

26. Mao L, Fan TF, Wu L, Yu GT, Deng WW, Chen L, et al. Selective blockade of B7$\mathrm{H} 3$ enhances antitumour immune activity by reducing immature myeloid cells in head and neck squamous cell carcinoma. J Cell Mol Med. 2017;21:2199-210.

27. Picarda E, Ohaegbulam KC, Zang X. Molecular pathways: targeting B7H3 (CD276) for human cancer immunotherapy. Clin Cancer Res. 2016; 22:3425-31. 
28. Kazarian M, Laird-Offringa IA. Small-cell lung cancer-associated autoantibodies: potential applications to cancer diagnosis, early detection and therapy. Mol Cancer. 2011;10:33.

29. Bhatia A, Kumar Y. Cellular and molecular mechanisms in cancer immune escape: a comprehensive review. Expert Rev Clin Immunol. 2014;10(1):41-62.

30. Bonanno L, Pavan A, Dieci MV, Di Liso E, Schiavon M, Comacchio G, et al. The role of immune microenvironment in small-cell lung cancer: distribution of PD-L1 expression and prognostic role of FOXP3-positive tumour infiltrating lymphocytes. Eur J Cancer. 2018;101:191-200.

31. Fridman $W H$, Pagès $F$, Sautès-Fridman $C$, Galon J. The immune contexture in human tumours: impact on clinical outcome. Nat Rev Cancer. 2012;12(4):298-306.

- fast, convenient online submission

- thorough peer review by experienced researchers in your field

- rapid publication on acceptance

- support for research data, including large and complex data types

- gold Open Access which fosters wider collaboration and increased citations

- maximum visibility for your research: over $100 \mathrm{M}$ website views per year

At BMC, research is always in progress. 